\title{
Conditional Survival and Annual Hazard Estimates of Classical Hodgkin Lymphoma
}

\author{
Reyizha Nuersulitan' \\ Mingzi Yang' \\ Lan $\mathrm{Mi}^{\prime}$ \\ Meng $\mathrm{Wu}^{\prime}$ \\ Xin Liu $^{2}$ \\ Lingyan Ping ${ }^{\prime}$ \\ Yan Xie ${ }^{\prime}$ \\ Weiping Liu' \\ Yuqin Song' \\ Jun Zhu'
}

'Key Laboratory of Carcinogenesis and Translational Research (Ministry of Education), Department of Lymphoma, Peking University Cancer Hospital \& Institute, Beijing, I00I42, People's

Republic of China; ${ }^{2}$ State Key Laboratory of Molecular Oncology and Department of Radiation Oncology, National Cancer Center/Cancer Hospital, Chinese Academy of Medical Sciences (CAMS) and Peking Union Medical College (PUMC), Beijing, I0002I, People's Republic of China
Correspondence: Jun Zhu

Key Laboratory of Carcinogenesis and

Translational Research (Ministry of

Education), Department of Lymphoma,

Peking University Cancer Hospital \&

Institute, No. 52 Fucheng Road, Haidian

District, Beijing, I00I42, People's

Republic of China

Tel +86 10 88196109

Fax +861088196115

Email zhu-jun2017@outlook.com
Background: In the present study, we have tried to understand how the level of risk and survival probability changes over time for patients with classical Hodgkin's lymphoma by employing conditional survival and annual hazard as dynamic estimates of prognosis and survival.

Methods: This retrospective study reviewed the clinical data of patients with newly diagnosed classical Hodgkin's lymphoma admitted to Peking University Cancer Hospital between January 1, 2008, and December 31, 2017. Conditional survival and annual hazard rate were defined as the survival probability and yearly event rate, respectively, assuming that patients have survived for a defined time.

Results: A total of 384 patients were included (median age, 32 years; range, 6-77 years), of which 218 (56.8\%) patients had early-stage disease. The median follow-up time was 41.3 months. The 5-year conditional overall survival (COS) rates remained favorable and showed an increase from $89 \%$ at treatment to $94 \%$ at year 5, while the 5-year conditional failure-free survival (CFFS) rate increased from $70 \%$ at treatment to $96 \%$ at year 5 . The annual hazard of failure decreased from over $15 \%$ at diagnosis to less than $5 \%$ after 3 years. Early-stage patients had constantly lower annual estimates for hazard of death (range, 0-3.0\%) and failure (range, 0-14.3\%). However, the hazard of failure in advanced-stage patients decreased from $24.2 \%$ at diagnosis to below $8 \%$ after 3 years, whereas the hazard of death was always at relatively low levels. Patients with a high IPS risk score $(\geq 3)$ had significantly lower COS and CFFS during the first 4 years. Patients who received the BEACOPP regimen had better 5-year COS and 5-year CFFS than those who received the ABVD regimen.

Conclusion: The survival probability increased and hazard of failure decreased over time. Keywords: Hodgkin disease, conditional survival, failure-free survival, failure hazard, prognosis

\section{Introduction}

Lymphoma is a malignant hematological tumor that originates from the lymphoid tissues and nodes and is often roughly classified as Hodgkin lymphoma (HL) and nonHodgkin lymphoma. According to its histologic features, HL can be subclassified as classical Hodgkin lymphoma (cHL) and nodular lymphocyte-predominant Hodgkin lymphoma (NLPHL). ${ }^{1}$ According to disease staging and risk stratification, risk-adapted therapy is used for HL treatment. Early-stage (stage I/II) HL patients are usually administered therapy with combined modalities, including chemotherapy and subsequent radiotherapy. Advanced-stage (stage III/IV) HL patients are often treated with chemotherapy that is administered over a relatively longer course, instead of radiation therapy. After first-line polychemotherapy, high-dose chemotherapy (HDCT) followed 
by autologous stem cell transplantation (ASCT) is adopted as the standard care for patients with relapsed HL. ${ }^{2-4}$

The Global Burden of Disease Study 2016 reported that, worldwide, nearly 28,700 deaths were caused by HL. ${ }^{5}$ In the past few decades, the survival of HL patients has improved significantly. In a previous retrospective study conducted at our center that included 488 cases of HL diagnosed between January 1, 1996, and December 31, 2015, the 5-year overall survival (OS) was $79 \%$ in the cHL group: it was $89 \%$ in the early-stage patients and $72 \%$ in the advanced-stage patients. ${ }^{6}$ Recently, another study at our center on 115 relapsed/refractory cHL patients who underwent HDCT followed by ASCT showed that the 5-year progression-free survival (PFS) and OS after treatment were $53 \%$ and $78.7 \%$, respectively. These results imply that it is still possible to achieve long-term remission in patients with relapsed/refractory $\mathrm{HL}$ who undergo ASCT. ${ }^{7}$

The prognosis of patients with cHL is estimated from the date of diagnosis. In general, in patients who have survived for a while after diagnosis, the prognosis usually improves over time and the initial estimations become less relevant. ${ }^{8}$ It is well known that the probability of an increase in survival is associated with accumulated survival time. However, the traditional OS estimate provides only limited information about how the magnitude of risk changes over time. Furthermore, it is not clear to what extent the baseline risk factors hold prognostic value for patients who have completed their initial therapy. Conditional survival and annual hazard estimates might provide more clinical information about the prognosis and how it changes over time. These dynamic estimates have been applied to several malignant tumors before, ${ }^{9-11}$ but there are only a few studies on their value with regard to predicting prognosis and survival in cHL. Therefore, this study aims to conduct a dynamic estimation of longterm survival and annual hazard rate in cHL patients.

\section{Methods}

\section{Patients}

This retrospective study reviewed the clinical data of 384 newly diagnosed cHL patients. These patients were admitted to Peking University Cancer Hospital between 1st January 2008, and 31st December 2017. The key inclusion criteria were (1) a biopsy specimen of newly diagnosed cHL confirmed by the pathology department of Peking University Cancer Hospital and (2) no previous treatment for HL. The key exclusion criteria were (1) central nervous system involvement and (2) human immunodeficiency virus. Computer tomography (CT) of the neck, thorax, abdomen, and pelvis, or positron emission tomography (PET)/CT, was used for staging and response evaluation. Assessment of conditional survival and failure hazard was based on stages, risk categories, and choice of chemotherapy regimen. According to the definition of prognostic factors by the National Comprehensive Cancer Network guidelines, ${ }^{12}$ early-stage HL patients are often divided into two groupsthose with a favorable prognosis and those with an unfavorable prognosis. The International Prognostic Score (IPS), which is calculated based on seven variables, is used for evaluating the prognosis of advanced HL patients by stratifying them into low-IPS $(<3)$ or high-IPS $(\geq 3)$ risk categories. Every procedure in this report involving human participants was implemented according to the ethical standards of the institutional committee and was in keeping with the 1964 Declaration of Helsinki and its later amendments or comparable ethical standards.

\section{Treatment}

For early-stage patients with favorable factors, the standard treatment regimen was four cycles of ABVD combined with RT (radiotherapy) or 6 cycles of ABVD. Furthermore, $6-8$ cycles of ABVD \pm RT continued to be used as the standard chemotherapy regimen for patients with advanced-stage disease. For patients who were below 60 years old, the BEACOPP regimen was the main choice for early-stage patients with unfavorable factors $(\geq 3)$ and advanced-stage patients with a high IPS score $(\geq 3)$.

\section{Efficacy Evaluation}

The response towards treatment was assessed by CT or PET/CT after every two cycles of chemotherapy or $4-8$ weeks after the final chemotherapy. Assessment of treatment response was based on the 2014 Lugano classification. Overall response rate (ORR) was defined as the rate of complete response (CR) plus partial response (PR). Stable disease (SD) and progressive disease (PD) were considered to indicate no response.

\section{Statistical Analysis}

OS was measured as the period from the date of diagnosis to date of death due to any cause or latest follow-up. FFS (failure-free survival) was calculated from the date of treatment to the date of disease progression, relapse, or the date of death. Conditional survival (CS) is based on the concept of conditional probability, ${ }^{10,13}$ and conditional overall survival 
(COS) is defined as the probability of surviving for a certain period based on the number of survival years of a patient. Likewise, the probability of FFS for a certain period is dependent on the supposition of the patient's survival for a specified interval of time with no failure, and this is defined as conditional failure-free survival (CFFS) ${ }^{14-16}$ In order to estimate the cumulative survival rates, the Kaplan-Meier method together with the Log rank test were used. Annual risk hazard is defined as the total follow-up time accumulated by the patients at risk within a given number of years divided by the number of annual events. All statistical analyses were conducted using IBM Statistics SPSS 26.0 (IBM Inc., Chicago) together with $\mathrm{R}$ version 3.6.3 (R Foundation for Statistical Computing, New York).

\section{Results \\ Cohort Characteristics}

This study enrolled 384 patients with a mean age of 32 years (range, 6-77 years) and a median follow-up time of 41.3 months. The baseline characteristics of the patients and their disease characteristics can be found in Table1. In sum, 34 $(8.9 \%)$ patients were 60 years or older. Further, 66 (17.2\%) patients had an Eastern Cooperative Oncology Group (ECOG) score of $\geq 1$, and 284 (74.0\%) had elevated lactate dehydrogenase (LDH) levels. With regard to disease stage, 218 patients had stage I/II (early stage) disease, while the other 166 patients had stage III/IV (advanced stage) disease. Among the advanced-stage patients, 77 (46.4\%) had a high IPS score ( $\geq 3)$, and 95 (43.6\%) early-stage patients had unfavorable factors. With regard to ethnicity, 357 (93\%) patients were Han Chinese; 2.9\%, Manchu; 2.1\%, Hui; $1.8 \%$, Mongolian; and 1.2\%, Yi. Among 50 adolescent/ pediatric patients $(<21$ years) with a median age of 18 years (range, 6-20 years), $30(60 \%)$ patients had early-stage disease. The year-wise incidence according to age and gender is shown in Figure S1. For all the years reported, the highest proportion of patients was between 21 and 60 years of age.

\section{First-Line Therapy and Response}

In total, 330 (85.7\%) patients received the ABVD regimen and $54(14 \%)$ patients received the BEACOPP regimen. The median number of cycles was 6 (2-8 cycles) for the ABVD regimen. Further, 196 (89.5\%) early-stage patients received the ABVD regimen, of which 94 had favorable factors and 102 had unfavorable factors. The remaining 22 $(10.5 \%)$ early-stage patients with unfavorable factors
Table I Summary of Clinical Characteristics of Patients with Classical Hodgkin Lymphoma $(\mathrm{N}=384)$

\begin{tabular}{|c|c|}
\hline Characteristics & No. of Patients (\%) \\
\hline \multicolumn{2}{|l|}{ Gender } \\
\hline Male & $217(56.5)$ \\
\hline Female & $167(43.5)$ \\
\hline \multicolumn{2}{|l|}{ Ethnicity } \\
\hline Han nationality & $357(93)$ \\
\hline Non-Han nationality & $27(7)$ \\
\hline \multicolumn{2}{|l|}{ Age (years) } \\
\hline$\geq 60$ & $34(9.1)$ \\
\hline$<60$ & 350 (90.9) \\
\hline \multicolumn{2}{|l|}{ Stage } \\
\hline I & $35(9.1)$ \\
\hline II & $183(47.7)$ \\
\hline III & $75(19.5)$ \\
\hline IV & 91 (23.7) \\
\hline B symptoms & $133(34.6)$ \\
\hline \multicolumn{2}{|l|}{ ECOG score } \\
\hline$E C O G \geq I$ & $66(17.2)$ \\
\hline ECOG $<1$ & $318(82.8)$ \\
\hline \multicolumn{2}{|l|}{ Early stage } \\
\hline Favorable factors & $94(43.2)$ \\
\hline Unfavorable factors & $124(56.9)$ \\
\hline \multicolumn{2}{|l|}{ Advanced stage } \\
\hline Low IPS score $(<3)$ & $89(53.6)$ \\
\hline High IPS score $(\geq 3)$ & $77(46.4)$ \\
\hline \multicolumn{2}{|l|}{ Regimen } \\
\hline ABVD & $330(85.7)$ \\
\hline BEACOPP & $54(14.0)$ \\
\hline \multicolumn{2}{|l|}{ Response to initial therapy } \\
\hline CR & $274(7 \mid .4)$ \\
\hline PR & $71(18.5)$ \\
\hline No response & $39(10.2)$ \\
\hline
\end{tabular}

Abbreviations: ECOG, Eastern Cooperative Oncology Group; IPS, International Prognostic Score; ABVD, Doxorubicin, Bleomycin, Vinblastine (vincristine), Dacarbazine; BEACOPP, Bleomycin, Etoposide, Doxorubicin, Cyclophosphamide, Vincristine, Procarbazine, Prednisone; CR, Complete Remission; PR, Partial Remission.

received a median of 6 (4-8) cycles of the BEACOPP regimen. Among the patients with advanced-stage disease, 134 (80.7\%) received the ABVD regimen (median number of cycles: 6 [range, 2-8]): 50 patients received 6 cycles and 68 patients received 8 cycles. The remaining 32 advanced-stage patients received the BEACOPP regimen with a median of 7 cycles (range, 4-8 cycles). A total of 73 patients with early-stage disease received radiotherapy, 
and 15 patients with advanced-stage disease received radiotherapy.

The ORR and CR rate of the entire cohort were 93.4\% and $78.1 \%$, respectively. For early-stage patients receiving the ABVD regimen, the ORR and CR rate were $90.9 \%$ and $45.5 \%$ respectively. In contrast, for early-stage patients receiving the BEACOPP regimen, the ORR and $\mathrm{CR}$ rate were $100 \%$ and $50 \%$ respectively. For advanced-stage patients receiving the $A B V D$ regimen, the $O R R$ and $C R$ rate were $85.8 \%$ and $66.4 \%$ respectively. In contrast, for advanced-stage patients receiving the BEACOPP regimen, the ORR and CR rate were $84.4 \%$ and $68.8 \%$ respectively. Among 50 patients who were $<21$ years, 44 (88\%) received the ABVD regimen and $6(12 \%)$ received the BEACOPP regimen. In this group, the ORR and CR rate were $98 \%$ and $66 \%$ respectively, and the 5-year OS and FFS were $92 \%$ and $60 \%$ respectively.

\section{Salvage Therapy}

Second-line therapy was administered in 108 patients with relapse or refractory cHL: $79(73.1 \%)$ patients had primary refractory disease, and 29 (26.9\%) patients relapsed after first-line therapy. Of the 108 patients, 82 (75.9\%) received the DICE/ICE regimen (dexamethasone/ifosfamide/cisplatin/etoposide or ifosfamide/cisplatin/etoposide) as salvage therapy, $11(10.2 \%)$ received the ABVD or BEACOPP regimen, $12(11.1 \%)$ patients received the GemOx (gemcitabine and oxaliplatin) or GDP (gemcitabine, cisplatin, and dexamethasone) regimen, and $3(2.8 \%)$ patients received other chemotherapy regimens. Further, 60 (55.6\%) patients received ASCT.

\section{Conditional Survival and Annual Hazard Estimates Over Time}

With a median observation time of 41.3 months, the 5-year OS was $89 \%$ and FFS was $70 \%$. The 5 -year COS increased to $92 \%(95 \% \mathrm{CI}, 86-96 \%)$ for 3-year survivorship, 92\% (95\% CI, 81-97\%) for 4-year survivorship, and 94\% (95\% CI, 82-98\%) for 5-year survivorship. Furthermore, the 5-year CFFS increased to $85 \%(95 \%$ CI, 79-89\%) for 1-year survivorship, 91\% (95\% CI, 85.0-94\%) for 2-year survivorship, 94\% (95\% CI, 88 97\%) for 3-year survivorship, 92\% (95\% CI, 79-97\%) for 4-year survivorship, and 96\% (95\% CI, 76-99\%) for 5-year survivorship (Figure 1A, B and Table S1 Supplementary Material). Five-year CFFS had favorable increases compared to five-year COS in the first three years after treatment (Figure 1C). The annual hazard estimates of failure decreased from $15 \%$ in the first year to less than $5 \%$ after 3 years, but the annual hazard estimates of death were consistently low (death: range, $0-4.3 \%$ ) (Figure 1D).

Further analysis shows that early-stage patients had a higher 5-year OS and 5-year FFS than advanced-stage patients (5-year OS: $92 \%$ [95\% CI, 86-96\%] vs 85\% [95\% CI, 76-91\%], 5-year FFS: 80\% [95\% CI, 76-85\%] vs 59\% [95\% CI, 49-67\%]). Early-stage patients had excellent 5-year COS, while advanced-stage patients consistently had lower 5-year COS than the patients in early-stage disease. The 5-year CFFS of both the advanced and earlystage patients increased over time. However, advancedstage patients who were failure-free achieved comparable 5-year CFFS to early-stage patients at year 4 (Figure 2A, $\mathrm{B}$ and Table S1 in the Supplementary Material). Earlystage patients had relatively stable annual death hazard estimates (death: range, 0-3.0\%), while advanced-stage patients consistently had superior death hazard estimates to early-stage patients (death: range: 0-6.3\%). Both earlystage and advanced-stage patients had continuously decreasing failure hazard estimates: the annual failure hazard estimates of early-stage patients were close to $8 \%$ (failure: range, $0-14.3 \%$ ) in the first year and then decreased to less than 3\% after 3 years. Similarly, the annual failure hazard estimates of advanced-stage patients were highest (failure: range, $0-24.2 \%$ ) in the first year and then decreased to less than $8 \%$ after 3 years (Figure 2C and D).

\section{Conditional Survival and Annual Hazard Estimates Stratified by Treatment Response}

A total of 274 (71.4\%) patients achieved complete remission (CR), 71 (18.5\%) patients achieved partial remission (PR), and 39 (10.2\%) patients did not respond. Patients with $\mathrm{CR}$ had better 5-year COS than the PR and noresponse groups in the first three years. At year 4, in patients with PR, the 5-year COS increased to $91 \%$ (95\% CI, 73-97\%), while in patients with no response, the 5 -year COS increased to $88 \%$ (95\% CI, 39-98\%). The 4-year values in the PR and no-responses groups were comparable to those of patients with CR. Patients with CR had consistently lower death hazards than those of the other two groups. Additionally, patients with no response exhibited a late increase in death hazard rates 


\section{A cos}

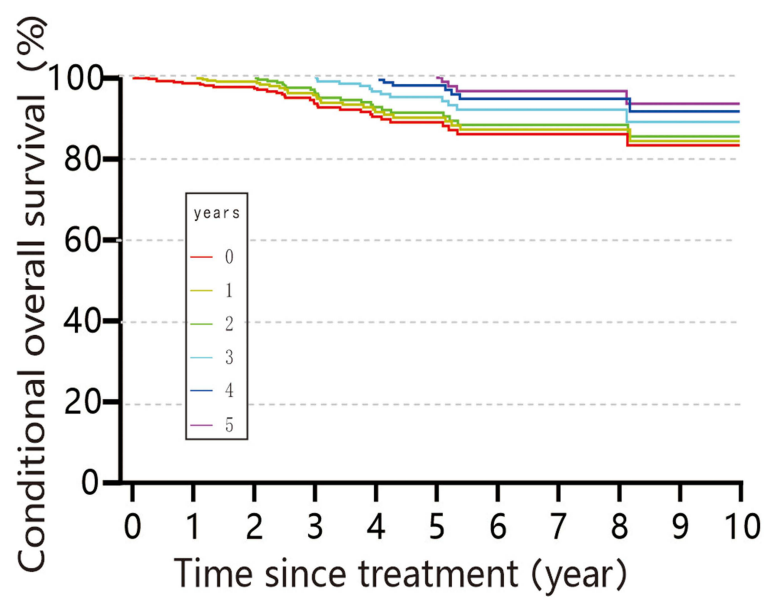

No.at risk

\section{B CFFS}

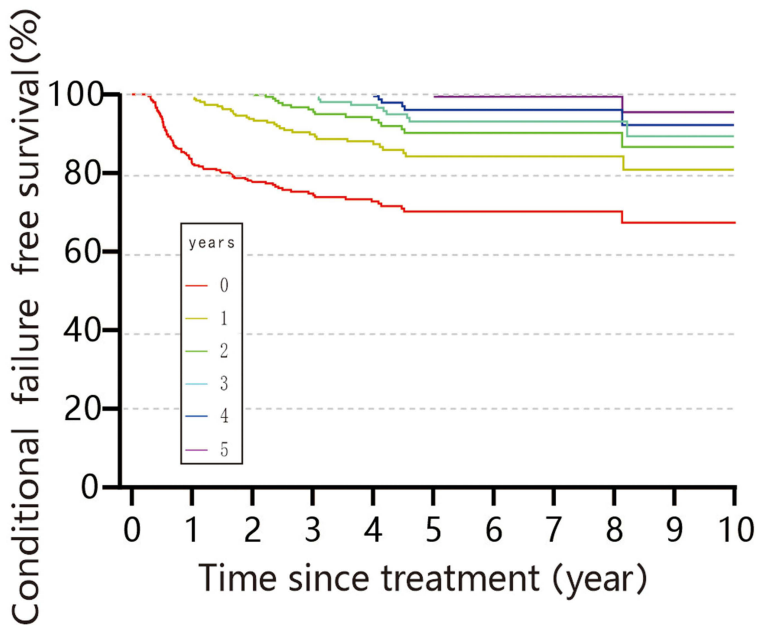

No.at risk $\begin{array}{llllll}384 & 278 & 147 & 68 & 32 & 4\end{array}$

\section{5-year CS}

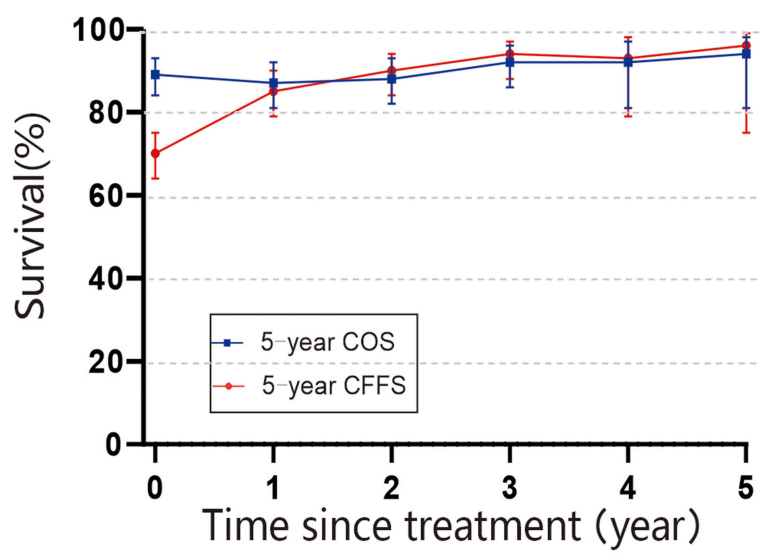

No.at risk

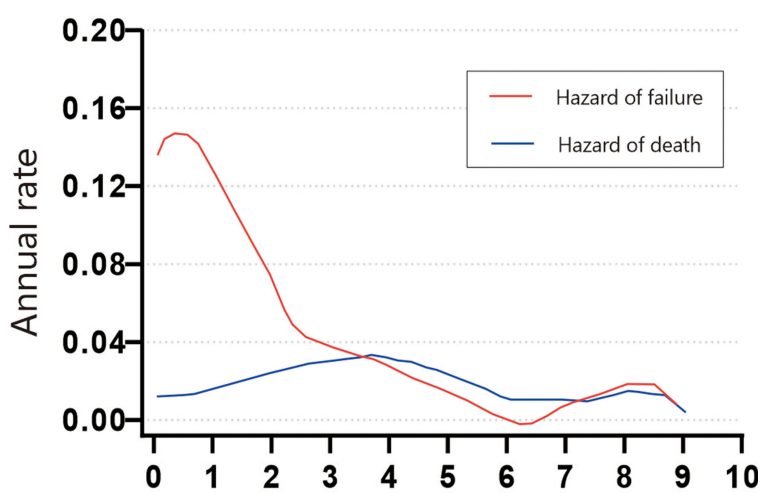

No.at risk

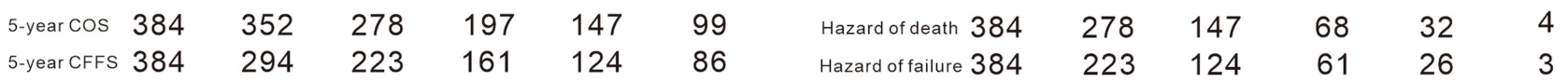

Figure I Conditional survival and annual hazard estimates of death and failure for classical Hodgkin lymphoma patients $(\mathrm{N}=384)$. (A) COS or conditional overall survival is a function of the number of surviving years. (B) CFFS or conditional failure-free survival is a function of the number of failure-free years. The differently colored lines represent the years of survival and failure-free survival after treatment, ranging from 0 to 5 years. (C) The probability of 5-year conditional survival is a function of the number of surviving or failure-free years since treatment (Error bars denote $95 \% \mathrm{Cls}$ ). (D) Smoothed plots of the annual estimates of death hazard and failure hazard according to time after treatment. 


\section{A 5-year COS}

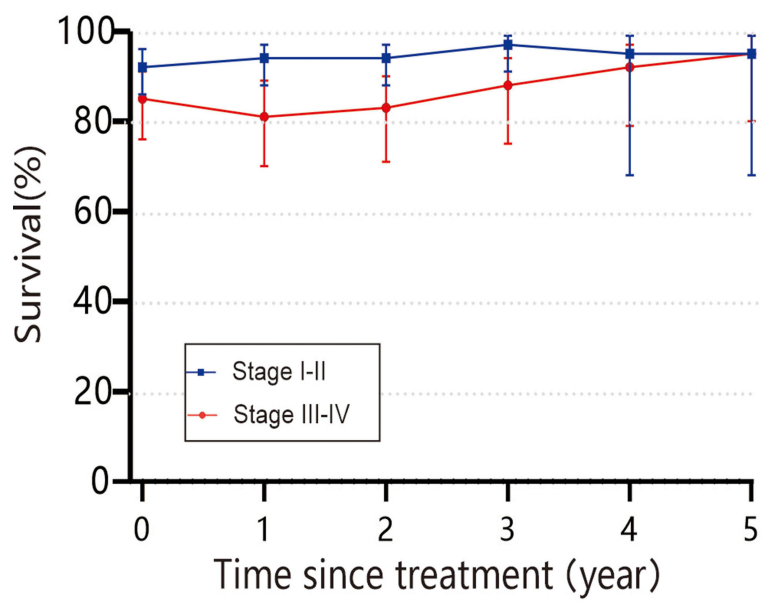

No.at risk

\begin{tabular}{|c|c|c|c|c|}
\hline Stage I-II 218 & 198 & 160 & 117 & 81 \\
\hline Stage III-IV 166 & 154 & 116 & 80 & 6 \\
\hline
\end{tabular}

C Hazard of death

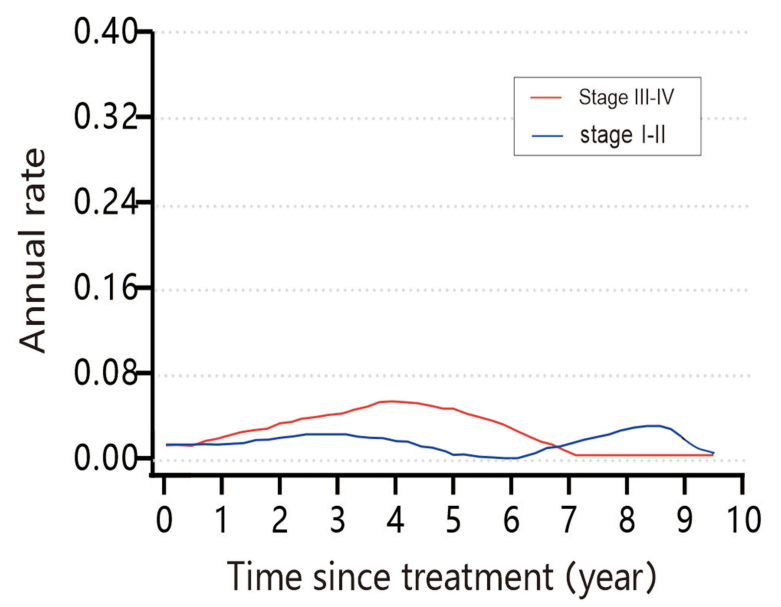

No.at risk

$\begin{array}{lllllll}\text { Stage I-II } & 218 & 160 & 81 & 41 & 20 & 2 \\ \text { Stage III-IV } & 166 & 118 & 66 & 27 & 12 & 2\end{array}$

\section{B 5-year CFFS}

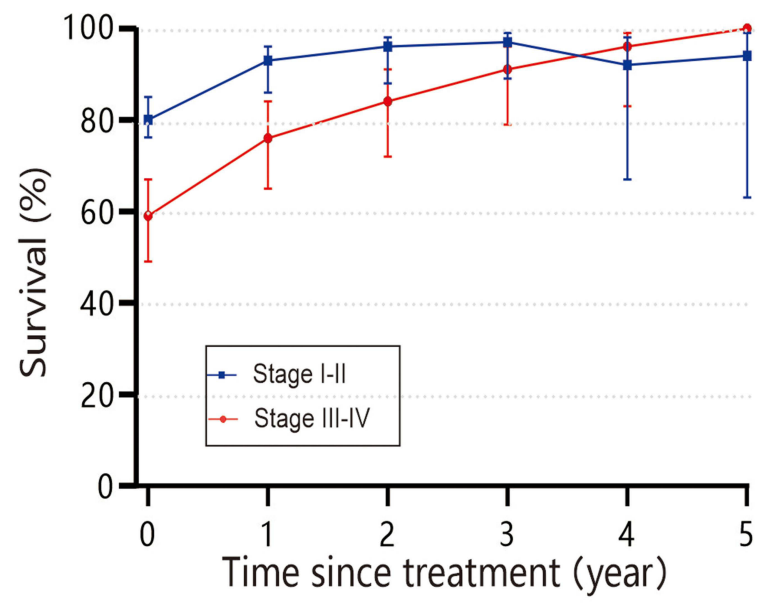

No.at risk

\begin{tabular}{|c|c|c|c|c|}
\hline Stage I-II 218 & 174 & 135 & 100 & 73 \\
\hline Stage III-IV 166 & 119 & 86 & 61 & 51 \\
\hline
\end{tabular}

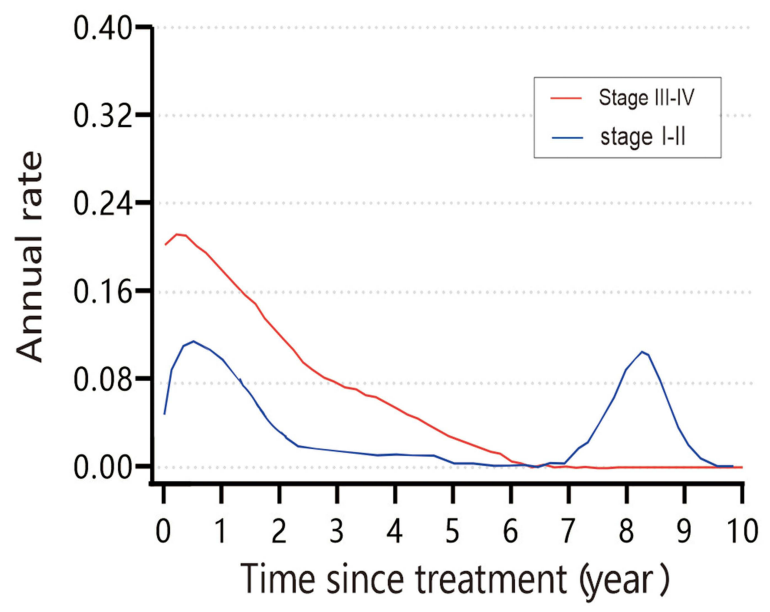

No.at risk

$\begin{array}{lcccccc}\text { Stage I-II } & 218 & 135 & 73 & 37 & 17 & 2 \\ \text { Stage IIIIV } 166 & 88 & 51 & 24 & 9 & 1\end{array}$

Figure 2 Conditional survival and hazard estimates of early-stage patients $(n=218)$ and advanced-stage patients $(n=166)$ with classical Hodgkin lymphoma. $(\mathbf{A}) 5$-year COS is the 5-year conditional overall survival over time of both early-stage and advanced-stage patients. (B) 5-year CFFS is the 5-year conditional failure-free survival over time of both early-stage and advanced-stage patients. The lines linearly connect different estimates (Error bars denote $95 \%$ Cls). (C and D) Smoothed plots of hazard estimates of death over time (C) and failure over time (D) for early-stage and advanced-stage patients. 
after year 3 (Figure S2 and Table S1 in Supplementary Material).

\section{Risk-Dependent Conditional Survival and Annual Hazard Estimates Over Time}

According to the staging system of the NCCN, early-stage HL patients were sorted into the early unfavorable stage and early favorable stage. Early-stage patients with unfavorable factors consistently had inferior 5-year COS to patients with favorable factors at any given time point. Early-stage patients with unfavorable factors and without failure beyond 2 years attained similar 5-year CFFS as early-stage patients with favorable factors (Figure 3A, $\mathrm{B}$ and Table S1 in the Supplementary Material). Patients with favorable factors had a lower risk hazard: death hazard was less than $1.5 \%$ (death: range, $0-1.4 \%$ ) and failure hazard was less than 5\% (failure: range, $0-4.8 \%$ ). Accordingly, patients with unfavorable factors initially had higher death hazard estimates, but they declined to less than $3.5 \%$ after 3 years (Figure $3 \mathrm{C}$ and D).

When the low IPS risk patients were compared with the high IPS risk $(\geq 3)$ patients, the former were found to have remarkably higher COS and CFFS (Figure 4A, B and Table $\underline{\mathrm{S} 1}$ in Supplementary Material). Compared with advancedstage patients who had a low IPS risk score, the patients who had a high IPS risk score $(\geq 3)$ had a higher initial risk. More specifically, in the high-risk patients, the annual hazard estimates were above $20 \%$ (failure: range, $0-23 \%$ ) for failure at the beginning, but they decreased to less than $12 \%$ at year 3 (failure: range, 0-11.7\%) (Figure 4C and D).

\section{Conditional Survival and Annual Hazards Stratified by Treatment}

Patients who received the BEACOPP regimen had better 5-year COS and 5-year CFFS than patients who received the ABVD regimen. In advanced-stage patients who received the BEACOPP regimen, the high IPS group achieved similar 5-year CFFS as the low IPS group after year 2 (Figure S3 in Supplementary Material).

\section{Discussion}

For clinicians and researchers, evaluating a patient's prognosis after initial treatment is crucial. Additionally, patients also need to be provided with accurate risk estimates. In the present study, we have tried to understand how the level of risk and survival probability changes over time for patients with cHL. To this end, we adopted an approach based on conditional survival, as it could be an alternative way for estimating long-term prognosis, as survival time elapses.

In this study, overall, the 5-year OS was $89 \%$ and the 5 -year FFS was $70 \%$. The 5-year COS exhibited little volatility, whereas the 5-year CFFS increased slightly from $70 \%$ to $96 \%$. It has been reported previously that nearly threefourth of relapses occurred in the first 2 years, and event-free survival (EFS) at year 2 is often correlated with lower hazard estimates and better outcomes..$^{14,17-19}$ Correspondingly, in our study, the annual hazard estimates of failure were relatively high at the beginning and decreased to less than 5\% after 3 years. Thus, these findings are in keeping with the studies mentioned above. Patients who could not achieve CR had poor 5-year OS compared to patients who achieved CR at the end of first-line chemotherapy. However, a spectacular improvement in 5-year COS was observed in the PR and noresponse patients with time. This result indicates that although patients who cannot achieve CR may have inferior survival initially, their survival is likely to improve with time. There are indeed some confounders, since some of patients who did not achieve CR received second-line therapies, including the DICE/ICE regimen and other salvage regimens or ASCT, which might have contributed to improving the 5-year FFS.

Previous studies ${ }^{20}$ have indicated that early-stage patients had superior survival rates to advanced-stage patients. Furthermore, there is evidence that a longer duration of survival from the time of disease onset is related to an increase in the probability of further survival. ${ }^{21}$ In our study, we found that although early-stage patients had better 5-year COS, advanced-stage patients achieved comparable 5-year CFFS at year 4. This result indicates that advanced-stage patients might have a slight but continuous improvement in conditional survival that increases over time. Additionally, advanced-stage patients initially had higher failure hazards that decreased to a level that was comparable to the initial failure hazards of early-stage patients. This finding indicates that longer survival or remission is often related to a higher probability of further survival or remission.

In our study, at year 2, early-stage patients with unfavorable factors achieved similar 5-year CFFS as early-stage patients with favorable factors. Furthermore, conditional survival was lower in high IPS risk patients than in low IPS risk patients but increased significantly with time. This trend indicates that patients with high IPS risk may have chances of achieving similar 5-year conditional survival as patients with low IPS risk, provided there is a sufficient amount of follow- 
A 5-year cos of early-stage patients

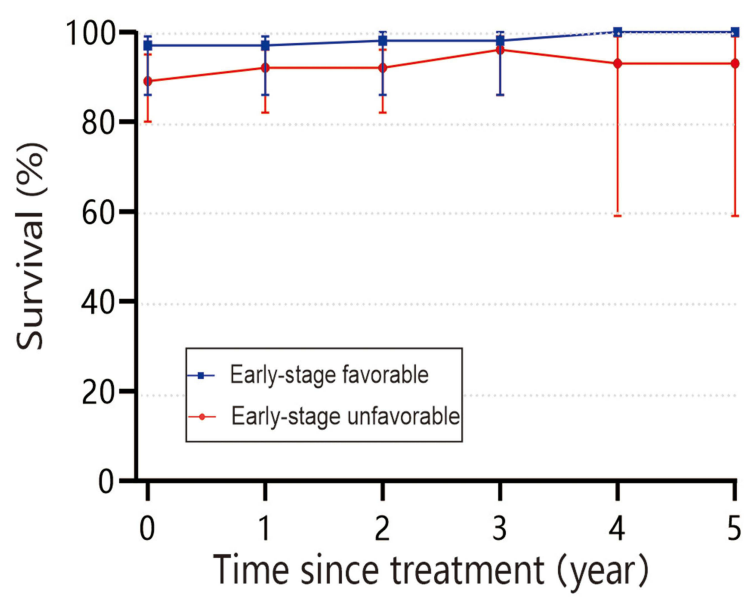

No.at risk

$\begin{array}{lcccccc}\text { favorable } & 94 & 86 & 66 & 48 & 35 & 23 \\ \text { unfavorable } 124 & 112 & 94 & 69 & 46 & 34\end{array}$

C Death hazards of early-stage patients

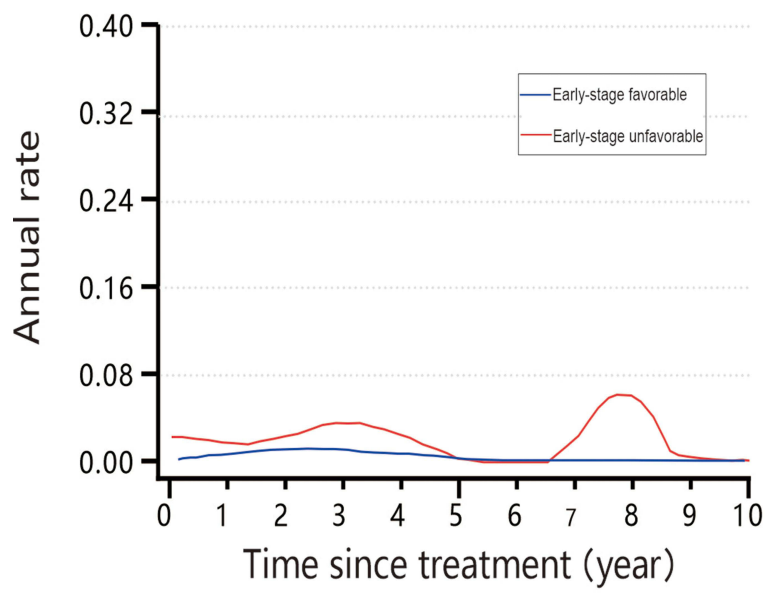

No.at risk

$\begin{array}{lcccccc}\text { favorable } & 94 & 66 & 35 & 16 & 5 & 1 \\ \text { unfavorable } 124 & 94 & 46 & 25 & 15 & 1\end{array}$

B 5-year CFFS of early-stage patients

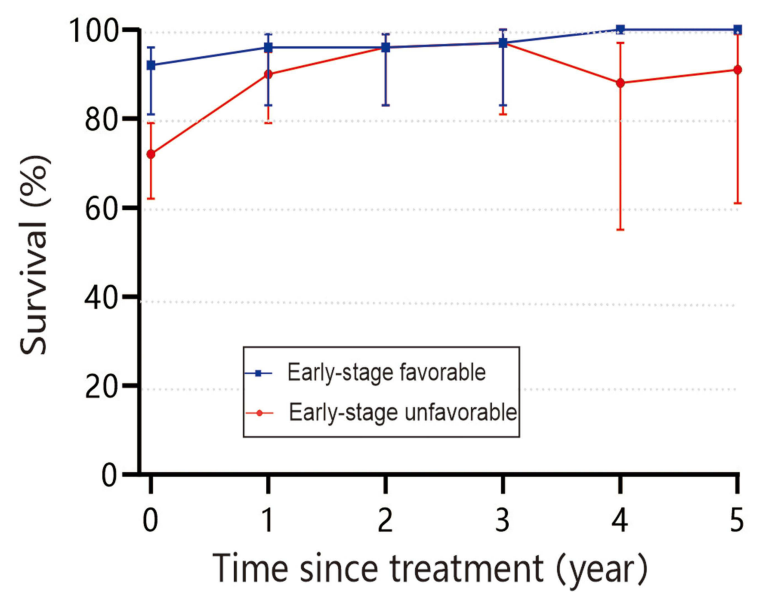

No.at risk

$\begin{array}{llllll}\text { favorable } 94 & 82 & 63 & 46 & 34 & 23 \\ \text { unfavorable124 } & 92 & 72 & 54 & 39 & 29\end{array}$

D Failure hazards of early-stage patients

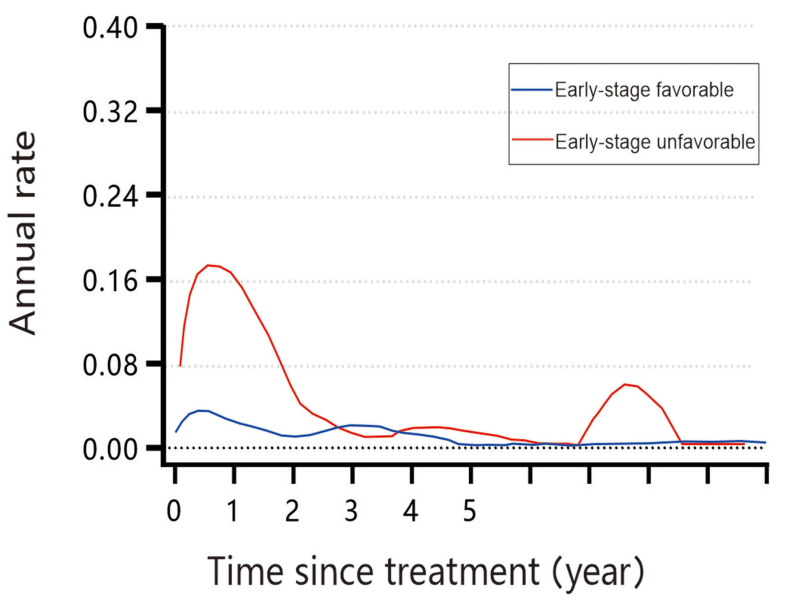

No.at risk

$\begin{array}{lcccccc}\text { favorable } & 94 & 63 & 34 & 16 & 5 & 1 \\ \text { unfavorable } 124 & 72 & 39 & 21 & 12 & 1\end{array}$

Figure 3 Conditional survival and hazard estimates stratified by risk groups in early-stage patients $(n=218)$. (A) 5 -year $\operatorname{COS}$ is the 5 -year conditional overall survival of early-stage patients with unfavorable and favorable factors over time. (B) 5-year CFFS is the 5-year conditional failure-free survival of early-stage patients with unfavorable and favorable factors over time (Error bars denote $95 \%$ Cls). (C and D) Smoothed plots of hazard estimates of death over time (C) and failure over time (D) for early-stage patients with favorable and unfavorable factors. 
A 5-year cos of advanced-stage patients

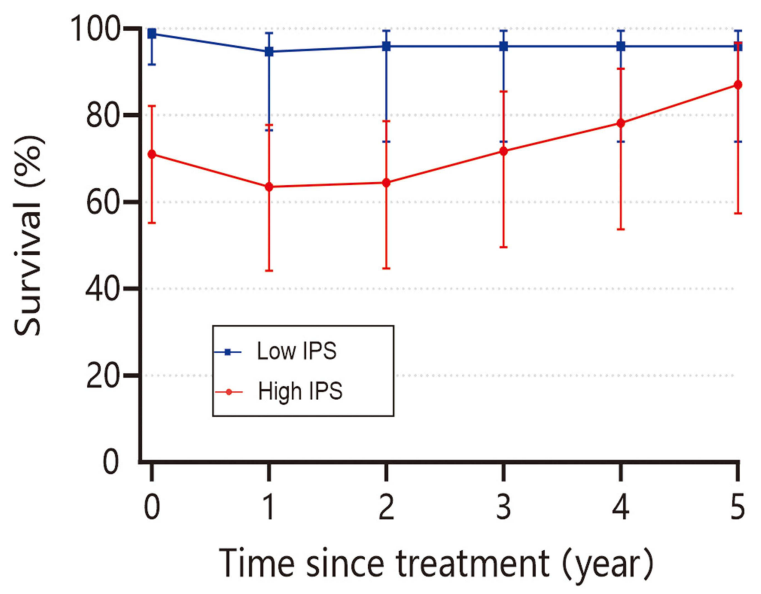

No.at risk

$\begin{array}{lllllll}\text { Low IPS } & 89 & 83 & 60 & 44 & 36 & 24 \\ \text { High IPS } & 77 & 71 & 56 & 36 & 30 & 18\end{array}$

C Death hazards of advanced-stage patients

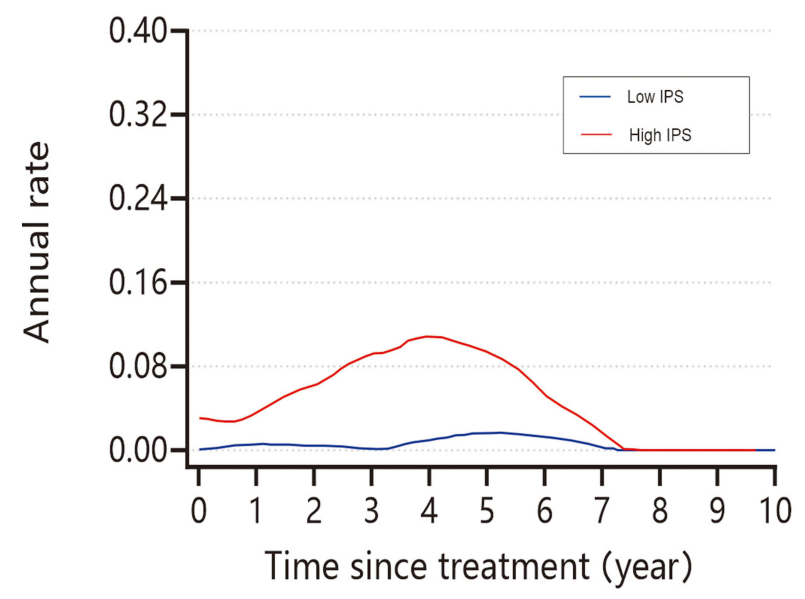

No.at risk

$\begin{array}{lllllll}\text { Low IPS } & 89 & 60 & 36 & 17 & 8 & 2 \\ \text { High IPS } & 77 & 56 & 30 & 10 & 7 & \end{array}$

B 5-year CFFS of advanced-stage patients

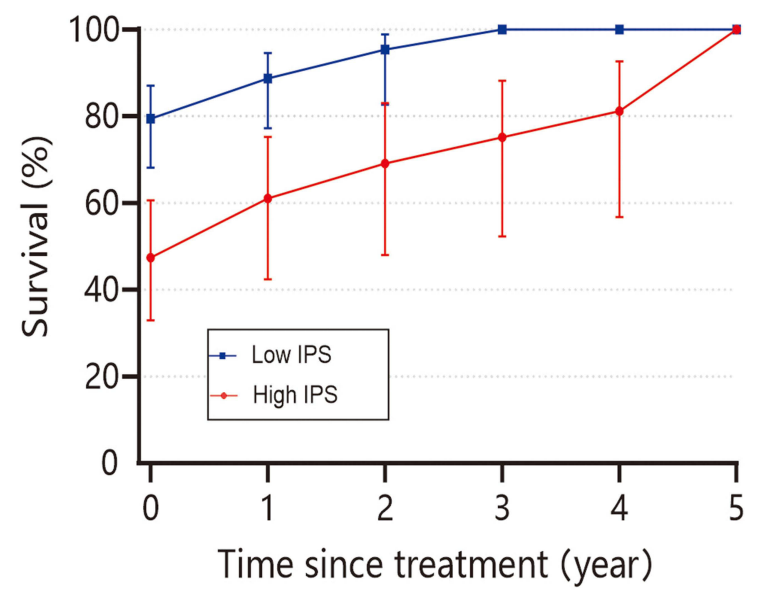

No.at risk

$\begin{array}{lllllll}\text { Low IPS } & 89 & 68 & 49 & 36 & 31 & 22 \\ \text { High IPS } & 77 & 51 & 37 & 25 & 20 & 12\end{array}$

D Failure hazards of advanced-stage patients

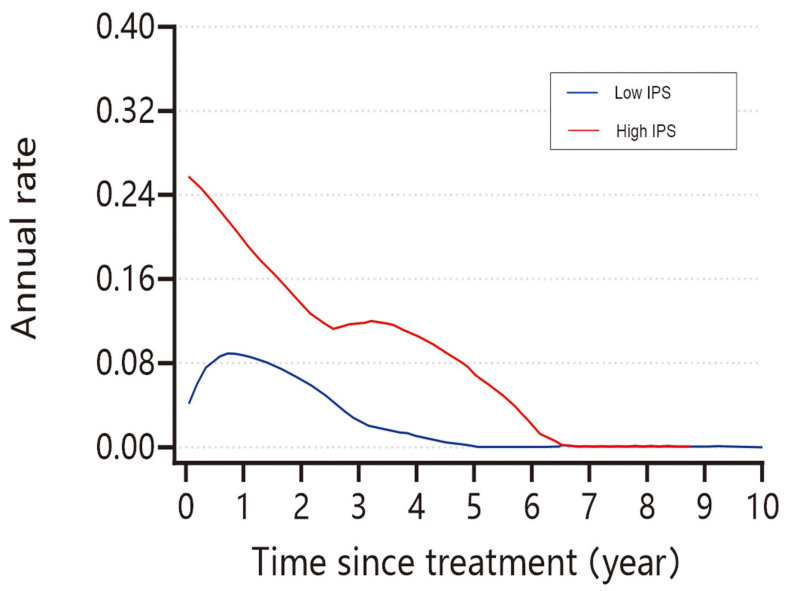

No.at risk

$\begin{array}{llllcll}\text { Low IPS } & 89 & 49 & 31 & 16 & 4 & 1 \\ \text { High IPS } & 77 & 37 & 20 & 8 & 5 & \end{array}$

Figure 4 Conditional survival and hazard estimates stratified by risk groups in advanced-stage patients $(\mathrm{n}=166)$. (A) 5-year COS is the 5 -year conditional overall survival over time in advanced-stage patients with low IPS score and high IPS scores. (B) 5-year CFFS is the 5-year conditional failure-free survival over time in advanced-stage patients with low IPS score and high IPS scores. The lines linearly connect different estimates (Error bars denote $95 \%$ Cls). (C and D) Smoothed plots for the hazard estimates of death over time in advanced-stage patients who had low IPS scores and high IPS scores (C), and smoothed plots for the hazard estimates of failure over time in advanced-stage patients who had low IPS scores and high IPS scores (D). 
up time. This also demonstrates that the IPS score loses its prognostic value over time, especially in patients who received the BEACOPP regimen. In each of the groups, according to our observation, the hazard estimates of failure were high during the first 2-3 years and remained relatively stable thereafter. This indicates that patients with HL should be followed up closely during the first 2-3 years after treatment.

The German Hodgkin Study Group (GHSG) HD14 trial ${ }^{22}$ reported that two cycles of escalated BEACOPP therapy followed by two cycles of ABVD $(2+2)$ treatment and then involved field radiation therapy (IFRT) remarkably ameliorates tumor control in early-stage cHL patients with unfavorable factors as compared with four cycles of ABVD, but it is also associated with more acute toxicity. In our study, we compare two different regimens (ABVD vs BEACOPP) in patients with early-stage unfavorable factors and those with advanced-stage disease. Although it seems that better conditional survival and lower hazard estimates are obtained in patients treated with BEACOPP, due to the small sample size of patients treated with BEACOPP, we cannot confirm that BEACOPP has more benefits, and further comparative verification with a larger sample is needed. Other studies reveal that patients treated with BEACOPP may have better PFS. ${ }^{23}$ However, when BEACOPP was combined with salvage therapy followed by ASCT, there was no significant difference in freedom from second progression and OS between the two regimens mentioned above. ${ }^{24}$ Taken together, because of the severe toxicity, especially reproductive toxicity, of the BEACOPP regimen and poor tolerance towards this therapy in the Chinese HL population, ABVD is the more popular choice in China for the treatment of $\mathrm{cHL}$.

\section{Limitations}

A main limitation is the retrospective nature of this study, as a result of which a selection bias was inevitable. The relatively short follow-up time is another limitation that might explain the relatively low number of long-term complications and influence the prognosis. Finally, further external validation is required to improve the generalizability of this study's findings.

\section{Conclusions}

The present findings indicate that compared with the conventional survival and hazard rates estimated from the diagnosis time, the probabilities of conditional survival and annual hazard rates provide more accurate and dynamic prognostic information on disease processes in patients with cHL.

\section{Data Sharing Statement}

The data that support the findings of this study are available from the corresponding author upon reasonable request.

\section{Ethics Statement}

This study's retrospective protocol was approved by the Ethics Committee at Peking University Cancer Hospital and Institute and the participating centers' institutional review boards and complied with the Declaration of Helsinki. The requirement for informed consent was waived based on the use of a deidentified data set. We have mentioned this in the revised manuscript.

\section{Acknowledgments}

The authors wish to thank the participating patients, hematologist-oncologists, pathologists, and statisticians for their invaluable contributions to this study.

\section{Author Contributions}

All authors contributed to data analysis, drafting, and revision of the article, have agreed on the journal to which the article will be submitted, gave final approval of the version to be published, and agree to be accountable for all aspects of the work.

\section{Funding}

This study was supported by the National Nature Science Foundation of China (grant numbers 81870154, 81972807, 81670187, 81970179, and 81700197), Beijing Natural Science Foundation (grant numbers 7202025 and 7202026), Capital's Funds for Health Improvement and Research (grant number 2018-1-2151), Beijing Municipal Science \& Technology Commission (grant number Z181100001918019), and Beijing Municipal Administration of Hospitals' Ascent Plan (grant number DFL20151001).

\section{Disclosure}

The authors declare that the research was conducted in the absence of any commercial or financial relationships that could be construed as a potential conflict of interest.

\section{References}

1. Cazzola M. Introduction to a review series: the 2016 revision of the WHO classification of tumors of hematopoietic and lymphoid tissues. Blood. 2016;127(20):2361-2364. doi:10.1182/blood-2016-03-657379 
2. Torok JA, Wu Y, Chino J, et al. Chemotherapy or combined modality therapy for early-stage hodgkin lymphoma. Anticancer Res. 2018;38 (5):2875-2881

3. Eich HT, Diehl V, Görgen H, et al. Intensified chemotherapy and dose-reduced involved-field radiotherapy in patients with early unfavorable Hodgkin's lymphoma: final analysis of the german hodgkin study group HD11 trial. J Clin Oncol. 2010;28(27):4199-4206. doi:10.1200/JCO.2010.29.8018

4. Engert A, Schiller P, Josting A, et al. Involved-field radiotherapy is equally effective and less toxic compared with extended-field radiotherapy after four cycles of chemotherapy in patients with early-stage unfavorable Hodgkin's lymphoma: results of the HD8 trial of the German Hodgkin's lymph. J Clin Oncol. 2003;21(19):3601-3608. doi:10.1200/JCO.2003.03.023

5. Naghavi M, Abajobir AA, Abbafati C, et al. Global, regional, and national age-sex specifc mortality for 264 causes of death, 1980 2016: a systematic analysis for the Global Burden of Disease Study 2016. Lancet. 2017;390(10100):1151-1210.

6. Liu W, Ji X, Song Y, et al. Improving survival of 3760 patients with lymphoma: experience of an academic center over two decades. Cancer Med. 2020;9(11):3765-3774. doi:10.1002/cam4.3037

7. Xie Y, Wang X, Leng X, et al. High-dose chemotherapy followed by autologous stem cell transplantation for patients with refractory/ relapsed classical Hodgkin lymphoma: a single center experience from China. Ann Hematol. 2020;99(3):549-555. doi:10.1007/ s00277-019-03812-w

8. Zabor EC, Gonen M, Chapman PB, Panageas KS. Dynamic prognostication using conditional survival estimates. Cancer. 2013;119 (20):3589-3592. doi:10.1002/cncr.28273

9. Anderson C, Smitherman AB, Nichols HB. Conditional relative survival among long-term survivors of adolescent and young adult cancers. Cancer. 2018;124(14):3037-3043. doi:10.1002/cncr.31529

10. Wang SJ, Emery R, Fuller CD, Kim JS, Sittig DF, Thomas CR. Conditional survival in gastric cancer: a SEER database analysis. Gastric Cancer. 2007;10(3):153-158. doi:10.1007/s10120-0070424-9

11. Lee JW, Ali B, Yoo HM, Park CH, Song KY. Conditional survival analysis in Korean patients with gastric cancer undergoing curative gastrectomy. BMC Cancer. 2015;15(1):1-7. doi:10.1186/s12885-0152022-2

12. Hoppe RT, Advani RH, Ai WZ, et al. Hodgkin lymphoma version 1.2017, NCCN clinical practice guidelines in oncology. J Natl Compr Cancer Netw. 2017;15(5):608-638. doi:10.6004/jnccn.2017.0064
13. Kim Y, Ejaz A, Spolverato G, et al. Conditional survival after surgical resection of gastric cancer: a multi-institutional analysis of the US Gastric Cancer Collaborative. Ann Surg Oncol. 2015;22 (2):557-564. doi:10.1245/s10434-014-4116-5

14. Hapgood G, Zheng Y, Sehn LH, et al. Evaluation of the risk of relapse in classical Hodgkin lymphoma at event-free survival time points and survival comparison with the general population in British Columbia. J Clin Oncol. 2016;34(21):2493-2500.

15. Hess KR, Levin VA. Getting more out of survival data by using the hazard function. Clin Cancer Res. 2014;20(6):1404-1409.

16. Pedersen NT, Christensen BE. Conditional survival of patients with diffuse large B-cell Lymphoma. Cancer. 2006;106:2165-2170.

17. Maurer MJ, Ellin F, Srour L, et al. International assessment of event-free survival at 24 months and subsequent survival in peripheral T-cell lymphoma. J Clin Oncol. 2017;35(36):4019-4026. doi:10.1200/JCO.2017.73.8195

18. Maurer MJ, Ghesquières H, Jais JP, et al. Event-free survival at 24 months is a robust end point for disease-related outcome in diffuse large B-cell lymphoma treated with immunochemotherapy. J Clin Oncol. 2014;32(10):1066-1073. doi:10.1200/JCO.2013.51.5866

19. Witzig TE, Hong F, Haioun C, et al. 24 months in patients with diffuse large B-cell lymphoma. Am J Hematol. 2016;91(2):179-184.

20. Wang YG, Zhao LY, Liu CQ, et al. Clinical characteristics and prognostic factors of primary gastric lymphoma: a retrospective study with 165 cases. Med (United States). 2016;95(31):1-6.

21. Liu X, Wu T, Zhu SY, et al. Risk-dependent conditional survival and failure hazard after radiotherapy for early-stage extranodal natural killer/T-cell Lymphoma. JAMA Netw open. 2019;2(3):e190194. doi:10.1001/jamanetworkopen.2019.0194

22. Von Tresckow B, Plütschow A, Fuchs M, et al. Dose-intensification in early unfavorable Hodgkin's lymphoma: final analysis of the German Hodgkin study group HD14 trial. J Clin Oncol. 2012;30 (9):907-913. doi:10.1200/JCO.2011.38.5807

23. Carde P, Karrasch M, Fortpied C, et al. Eight cycles of ABVD versus four cycles of BEACOPPescalated plus four cycles of BEACOPPbaseline in Stage III to IV, International Prognostic Score $\geq 3$, high-risk Hodgkin lymphoma: first results of the Phase III EORTC 20012 intergroup trial. J Clin Oncol. 2016;34 (17):2028-2036. doi:10.1200/JCO.2015.64.5648

24. Viviani S, Zinzani PL, Rambaldi A, et al. ABVD versus BEACOPP for Hodgkin's lymphoma when high-dose salvage is planned. New Eng J Med. 2011;365:203-212.

\section{Publish your work in this journal}

Cancer Management and Research is an international, peer-reviewed open access journal focusing on cancer research and the optimal use of preventative and integrated treatment interventions to achieve improved outcomes, enhanced survival and quality of life for the cancer patient.
The manuscript management system is completely online and includes a very quick and fair peer-review system, which is all easy to use. Visit http://www.dovepress.com/testimonials.php to read real quotes from published authors. 